\title{
Diferencias de composición corporal y condición fisica en educadoras de párvulo con normopeso, sobrepeso y obesidad: estudio transversal
}

\author{
Diffences of body max index, body \\ composition, and fitness in normo weight, \\ overweight and obese kindergarten \\ teachers: cross-sectional study
}

\section{ABSTRACT}

Objective: To describe body mass index (BMI), body composition, basal metabolic rate, physical condition and the relationship between them in Chilean kindergarten teachers. Subjects and Methods: 46 teachers (age 39,5 \pm 8,6) from the Bio Bio province(Chile) were weighed and heighted and BMI was measured. In addition, fat mass, lean and bone percentages, basal metabolic rate, muscular strength and cardiorespiratory fitness were measured. Results: The teachers had an average BMI of overweight $(26,7 \pm 3,5)$ and a high fat percentage $(35,1 \pm 5,9)$ according to national reference values. 58,7\%were overweight and moderately obese. When teachers were compared according $B M I$, teachers with obesity had on average a higher percentage of fat mass $(P=, 000)$, less lean mass $(P=, 000)$, took more time to complete the UKK test $(P=035)$ and had a lower vo2max $(P=, 001)$ than normal weight teachers. BMI had a negative association in relation to scores of the physical performance test. Conclusions: The overweight/obese teachers had more fat mass, reduced muscle mass and had worse results on fitness tests. Condition worrying thinking about the important role in modeling healthy habits to preschoolers.

Key words: body max index; body composition; physical fitness; faculty.
Rafael Zapata-Lamana (1) Matías Monsalves-Alvarez $(2,3)$

Igor Cigarroa Cuevas $(4,5)$

Mauricio Castro-Sepúlveda (6)

Gabriela Salazar Rodríguez (7)

Felipe Abad-Colil (8 2) Instituto de Nutrición y Tecnología de los Alimentos (INTA), Universidad de Chile. (3) Departamento de Ciencias Biológicas, Facultad de Ciencias Biológicas, Universidad Andrés Bello, Santiago, Chile. (4) Kinesiología, Facultad de Salud, Universidad Santo Tomás, Los Ángeles, Chile. (5) Departament de Psiquiatria i Medicina Legal, Institut de Neurociències, Universitat Autònoma de Barcelona, Barcelona, España. (6) Laboratorio de Ciencias del Ejercicio, Escuela de Kinesiología, Facultad de Medicina Universidad FinisTerrae, Santiago, Chile. (7) Laboratorio de Metabolismo Energético e Isótopos Estables, Instituto de Nutrición y Tecnología de los Alimentos y Nutrición, Universidad de Chile, Santiago, Chile. (8) Facultad de Ciencias de la Actividad Física, Universidad San Sebastián, Valdivia, Chile.

Dirigir la correspondencia a: Profesor Igor Cigarroa Cuevas, calle de Besa 22, ático 2, L'Hospitalet de Llobregat, 08904, Barcelona, España.

E-mail: icigarroa@santotomas.cl / igorcigarroa@yahoo.es

Este trabajo fue recibido el 17 de Marzo de 2016 y aceptado para ser publicado el 2 de Octubre de 2016.

\section{INTRODUCCIÓN}

La obesidad se ha incrementado de forma significativa en las últimas décadas y es considerada actualmente un problema de salud pública (1). Según la última encuesta nacional de salud en Chile las mujeres en promedio presentan 33\% de sobrepeso y $30,7 \%$ de obesidad que disminuye a medida que aumenta el nivel educativo, pero aumenta con la edad. Si además se adiciona que $92,9 \%$ de las mujeres indicó en la misma encuesta que realizan menos de 30 minutos 3 veces por semana de actividad física en su tiempo libre tenemos un gran porcentaje de la población femenina adulta sedentaria con malnutrición por exceso y con altas probabilidades de generar enfermedades crónicas no transmisibles(ECNTs)(2). Las causas del aumento de peso se han sido asociado a factores genéticos, psicológicos y conductuales tales como hábitos alimentarios, tiempo frente a las pantallas y nivel de actividad física (3). En 
relación a las causas conductuales, la condición física puede llegar a ser un importante predictor de mortalidad, la cual al ser mejorada es capaz de reducir los factores de riesgo de enfermedades independiente al peso corporal (4).

Los profesores y educadoras de párvulo, no son una excepción a las tendencias mundiales de sobrepeso y obesidad. Un estudio realizado en Alemania con una muestra de 313 educadoras de párvulo (18-62 años) observó que 41,2\% y $17,9 \%$ presentaban sobrepeso y obesidad respectivamente (5); prevalencia más alta comparada a valores de referencia de su país. En Chile, las educadoras de párvulos son las profesionales responsables de la educación en los preescolares, reciben una formación universitaria con conocimientos sobre estilos de vida saludable, que responden a los lineamientos que explicitan las bases curriculares de la educación parvularia, específicamente en el núcleo de autonomía y vida saludable (6).De esta forma, si se considera que los menores de 6 años permanecen gran parte de sus días en establecimientos educativos(5), las educadoras de párvulo tienen una gran influencia promoviendo y practicando hábitos de vida activa con sus alumnos, asumiendo un rol clave en la implementación de estrategias de prevención de la inactividad física y obesidad infantil(7). Su capacidad de promover el juego en los recreos, se asocia positivamente con un mejor rendimiento de patrones motores en los niños (8). Además, se ha demostrado que las educadoras pueden ser capaces de aumentar la intensidad de las actividades que los menores realizan (9) lo que promovería el incremento del gasto energético y la disminución de la obesidad (10).

Pese al importante rol que juegan las educadoras de párvulo en la creación de hábitos de salud y la mejoría de la capacidad física de los niños, no se conoce con certeza cómo es el IMC, composición corporal y la condición física de este grupo de profesionales. Son escasos los estudios internacionales que hayan evaluado estas variables en las educadoras de párvulo, no encontrando estudios nacionales quelas caractericen a nivel nutricional y físico. El objetivo del estudio fue determinar el IMC, la composición corporal, la tasa metabólica basal y la condición física de las educadoras de párvulo de la provincia de Bio-Bio, Chile.

\section{SUJETOS Y MÉTODOS}

Diseño: Estudio cuantitativo, transversal y de diseño ex post facto.

Participantes: del total de las educadoras del sistema municipal de educación de párvulo de la provincia del Bio-Bio (Chile) se evaluaron voluntariamente su IMC, composición corporal, tasa metabólica basal y condición física a 48 mujeres de entre 24 y 50 años, que representan $73,85 \%$ de las educadoras de la provincia, previa carta de consentimiento, autorización de un médico y cuestionario de aptitud para la actividad física (CAAF) completados satisfactoriamente. Tanto el control médico como el C-AAF indicaron la idoneidad cardiovascular de los sujetos para realizar los test. 2 participantes que tenían una patología osteo-articular aguda fueron eliminadas del estudio, por lo que la muestra quedo formada por 46 educadoras (tabla 1). El n muestral no alcanzó a ser representativa del total de las educadoras de párvulo del sistema municipal de la provincia de Bio-Bio ( $n=56$, considerando una heterogeneidad de $50 \%$, $5 \%$ de margen de error y $95 \%$ de nivel de confianza).

Procedimientos: Todas las evaluaciones se realizaron entre las 09:00 y 12:00 horas, durante 2 semanas, siempre en la misma habitación con condiciones estándar de humedad (50\%+-10) y temperatura $\left(21^{\circ} \mathrm{C}+-2\right)$.

Evaluaciones: Cuestionario de Aptitud para la Actividad
Física (C-AAF): Se utilizó la versión adaptada del original Activity Readiness Questionnaire (PAR-Q). Consta de 7 preguntas con respuesta "si" o "no" y permite identificar los riesgos antes de iniciar un programa de ejercicio físico. Es necesario responder "no" a todas las preguntas para poder iniciar el programa de ejercicio. Si se responde afirmativamente a alguna pregunta, se recomienda consultar a un médico.

Antropometría: Se evaluó la estatura y el peso mediante una balanza con tallímetro (Tanita, WB-3000, Japón) con sensibilidad de 0,1 cm y 0,1 kg respectivamente. Se calculó el índice de masa corporal (IMC), mediante la fórmula de Quetelet peso/ (estatura2) (11). Los valores de IMC fueron establecidos de acuerdo a los indicadores de la OMS para adultos sobre 20 años, donde normopeso: IMC entre 18,5 y 24,9, sobrepeso: IMC entre 25 y 29,9 y obesidad: IMC igual o superior a 30 (12). El equipo del proyecto fue previamente validado y estandarizado con la técnica antropométrica mediante método Stantro.

Composición corporal y tasa metabólica La medición del porcentaje de grasa, masa magra, masa ósea y tasa metabólica basal, se efectuó con las participantes descalzas en posición de bipedestación con los brazos relajados junto al tronco realizando una medición pie-pie mediante 4 electrodos (metatarso-calcáneo), a través de bioimpedanciometro (Tanita SC-331, Japón) con una sensibilidad de 1\% para porcentaje de grasa y 0,1\% para masa libre de grasa (13).

Condición física: Se evaluó mediante la fuerza explosiva y la capacidad cardiorrespiratoria.

a) Fuerza explosiva: fue evaluada mediante el test squat jump, el cual permite estimar mediante la altura alcanzada la capacidad de los miembros inferiores de manifestar fuerza explosiva (14). Se utilizó la plataforma de salto DM Jump by Prometheus, con la Formula Altura=Tiempo de Vuelo x 1,226. Cada participante tuvo 3 oportunidades de saltar, se utilizó para la estadística el promedio de los tres saltos. (Se corrigió a posteriori altura alcanzada/peso)(15).

b) Capacidad cardiorrespiratoria: se evaluó mediante el UKK Test, el cual consiste en realizar una caminata de 2 kilómetros en una superficie plana lo más rápido que pueda, no fue posible correr en ningún momento en la prueba. El resultado del test es considerado como un indicador de condición física, el que toma en atención la edad de la persona, sexo, altura, peso, tiempo en que se demora en completar los 2 kilómetros del test y la frecuencia cardíaca al término del test (16).Para aumentar la seguridad de la prueba, se valoró la percepción del esfuerzo utilizando el índice de Borg modificado.

Comité de ética: Las evaluaciones del IMC, composición corporal y capacidad física fueron aprobadas por el Comité de ética de la Universidad de Concepción, Los Ángeles, (Chile) y se realizaron previa firma de cartas de consentimiento, respetando la autonomía del sujeto según la ley 20.584. Todos los procedimientos utilizados para la realización del presente estudio han seguido los principios éticos para las investigaciones médicas en seres humanos según la Declaración de Helsinki actualizada en la $64^{a}$ Asamblea General, Fortaleza, Brasil, octubre 2013.

Análisis estadísticos: Los resultados fueron expresados como promedio \pm desviación estandar y en el análisis descriptivo de la muestra se agregaron los intervalos de confianza. Los datos se analizaron utilizando el programa SPSS (v.19.0, SPSS Inc., USA). Para determinar la normalidad de las variables analizadas se utilizó el test de Shapiro-Wilk y para determinar la homogeneidad de varianzas la prueba de Levene. Se usó ANOVA de un factor para determinar diferencias globales 
de los grupos y luego el análisis post hoc de Duncan para determinar las diferencias significativas entre cada uno de los grupos. Finalmente, se hizo un análisis de correlación bi variadas de Pearson ( $r$ ) ente todas las variables en estudio e IMC. Para determinar diferencias significativas se consideró ${ }^{*} p<0,05 y{ }^{* *} p<0,001$.

\section{RESULTADOS}

Las educadoras obtuvieron un IMC promedio de $26,7 \pm 0,5$, situándolas en un IMC de sobrepeso, valor similar $(26,8)$ al promedio nacional para mujeres con nivel educacional alto (universitario) (2). Además, un porcentaje de masa grasa

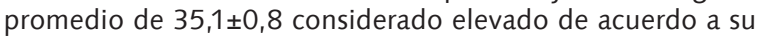
IMC y edad (12) (tabla 1). Al dividir la muestra por IMC, no existieron educadoras de párvulo con bajo peso u obesidad mórbida, 41,3\% estaban en normopeso, 41,3\% sobrepeso y $17,14 \%$ obesidad $(15,2 \%$ leve y $2,2 \%$ moderada). El porcentaje de sobrepeso fue superior $(32,0 \%)$ y el de obesidad inferior $(19,0 \%)$ al promedio nacional para mujeres con nivel educacional alto(2).Un 17,4\% de la muestra tenía porcentajes de masa grasa superiores al $40 \%$ y de masa magra menores a $56 \%$. Cuando se compararon a las educadoras por IMC se observó que las pertenecientes al grupo normopeso tenían menos porcentaje de masa grasa y una menor tasa metabólica basal que los grupos con sobrepeso y obesidad. Además, el grupo normopeso, presentó más masa muscular que el grupo con obesidad. En relación a la condición física, en el test de fuerza explosiva squat jump, al corregir la altura alcanzada/ peso, se observó que las educadoras con normopeso saltaron más que el grupo con obesidad, se demoraron menos tiempo en realizar el UKK test y con un mayor vo2max en comparación con el grupo con obesidad (tabla 2). Cuando se correlacionaron todas las variables dependientes versus el IMC y el porcentaje de masa grasa los resultados son prácticamente iguales. Particularmente, en relación al IMC, se evidenció una asociación positiva con el porcentaje de masa grasa y negativa con el porcentaje de masa magra, ósea y con los test de capacidad física (tabla 3).

\section{DISCUSIÓN}

La obesidad en Chile es un problema de salud pública creciente. Según el último informe de salud de la Organización para la cooperación y el desarrollo económico (OCDE) Chile posee el $6^{\circ}$ lugar de los países miembros con mayor cantidad de adultos con obesidad (17). Las educadoras de párvulo de la provincia del Bio-Bio evaluadas no son una excepción a la epidemiología nacional y presentaban altos niveles de sobrepeso $(41,3 \%)$ con promedios más altos que los niveles de referencia nacional para edad y nivel educativo (2). Estudios previos ya habían reportado en poblaciones específicas de trabajadores niveles más altos de IMC que valores de referencia nacional (18). Incluso estudios habían mostrado influencias específicas del género en algunas clases de ocupaciones en generar sobrepeso y obesidad (19). Hoffman (2013) al igual que los resultados de nuestro estudio encontró que los niveles de sobrepeso y obesidad de educadoras de párvulo estaban por sobre los valores de referencia nacional (20).

El IMC es un indicador confiable, económico y fácil para profesionales de la salud. Si bien, no mide directamente la grasa corporal, investigaciones han propuesto que tiene buena relación con mediciones directas de la grasa corporal como absorciometría dual de rayos $\times$ (DEXA) y con la bioimpedancia

TABLA 1

Descripción de las variables antropométricas, de composición corporal, metabólicas y de capacidad física.

Variables antropométricas

$\begin{array}{lll}\text { Edad (años) } & 39,5 \pm 8,6 & {[36,9-42,1]} \\ \text { Peso }(\mathrm{kg}) & 67,5 \pm 9,1 & {[64,7-70,2]} \\ \text { Talla }(\mathrm{m}) & 1,60 \pm 0,08 & {[1,57-1,62]} \\ \text { IMC }\left(\mathrm{kg} / \mathrm{m}^{2}\right) & 26,7 \pm 3,5 & {[25,6-27,8]}\end{array}$

Variables de composición corporal

Masa grasa (\%) $\quad 35,1 \pm 5,9$

$[33,3-36,8]$

Masa magra (\%)

$61,7 \pm 5,6$

$[60,1-63,4]$

Masa ósea (\%)

$3,3 \pm 0,3$

$[3,2-3,4]$

Tasa metabólica basal (kcal/dia)

$1328,9 \pm 106,3$

$[1297,4-1360,5]$

Variables de capacidad física

Test de fuerza explosiva Squat jump

Altura alcanzada $(\mathrm{cm})$

$14,0 \pm 3,6$

$0,21 \pm 0,06$

$1874,8 \pm 490,5$

$[12,9-15,0]$

$[0,20-0,23]$

Potencia necesaria (watts)

Test de capacidad aeróbica UKK test

FC Inicio del Test UKK (Ipm)

FC Final del Test UKK (Ipm)

$81 \pm 10,6$

$156,6 \pm 17,2$

Tiempo de prueba (min)

$18,9 \pm 1,2$

$26,7 \pm 4,7$

$[18,5-19,3]$
$[1729,1-2020,4]$

$[77,8-84,2]$

$[151,5-161,7]$

$[25,4-28,1]$

Los datos están presentados en media desviación estándar e intervalo de confianza $(n=46)$. 
(21). Las educadoras evaluadas se encontraron en un IMC de sobrepeso con riesgo de salud (22). Un estudio previo evaluó el IMC de educadoras de párvulos y profesores de enseñanza básica, en promedio los educadores presentaban un IMC de 27,1 y $25 \%$ de ellos se encontraban en estado de obesidad (23), resultados similares a los obtenidos en nuestro estudio. En este sentido, Hernández et al. (2010), evaluaron a 91 mujeres sanas no educadoras con edad $(34 \pm 7)$ e IMC $(25 \pm 2,9)$ de similares características etarias y étnicas a las profesionales de la educación evaluadas, encontrando que en promedio tenían un $38 \%$ de masa grasa (24); en nuestro estudio el porcentaje de masa grasa fue de $35 \%$. Si bien las educadoras de párvulo deben ser un modelo de referencia para los niños que educan en relación a estilos de vida saludable, estas presentaron un IMC y un \% de grasa similar a las mujeres no educadoras. Con respecto al gasto metabólico basal medido en nuestro estudio (1328,9+106,3 kcal/día), es un fiel reflejo de la composición corporal y los hábitos de ingesta de las evaluadas, Rosado et al. (2010) también mostró en un estudio realizado en 26 mujeres con sobrepeso y en su mayoría sedentarias un gasto metabólico basal similar al encontrado en este estudio (1488,58 $\pm 139,08 \mathrm{Kcal} /$ día) $(25)$.

Además, los resultados mostraron que cuando las educadoras fueron divididas en relación a su IMC, quienes se encontraban en el grupo de sobrepeso y obesidad moderada presentaban peor condición física que las educadoras con IMC de normopeso. En concordancia con este hallazgo el \% de masa grasa se asoció negativamente con el rendimiento en el test de fuerza $(r=-0,49 ; p<0,001)$, de capacidad aeróbica $(r=0,31$; $p<0,05)$ y con el Vo2max $(r=-0,43 ; p<0,001)$. Nuestros resultados son consistentes con una gran cantidad de estudios transversales hechos tanto en niños y adolescentes (26) como en adultos (27) que indican que los niveles de condición física se asocian con numerosos parámetros de salud, entre ellos el IMC, el porcentaje de grasa y la masa muscular. Ortega FB et al (2010) en un estudio hecho en adolescentes $(n=1075)$ mostró una asociación inversa entre la capacidad aeróbica y la circunferencia de cintura $(p<0,001)(28)$. El fomento e incremento de los niveles de actividad física, por un lado, y/o la mejora de la condición física, por otro, pueden tener un papel fundamental en la prevención del sobrepeso y de la obesidad no sólo durante la propia infancia y adolescencia sino incluso años más tarde (29). Además, se relacionan con una menor prevalencia de síndrome metabólico, enfermedades osteomusculares y una mejor calidad de vida en la adultez (30).

En relación a las pruebas de capacidad física, se evaluó la fuerza explosiva de las extremidades inferiores a través de la altura de salto vertical corregida por peso para evitar ventajas producidas por la masa. Las educadoras con obesidad saltaron menos que las que tenían un peso normal. La baja fuerza explosiva en el tren inferior podría limitar el movimiento y desplazamiento de las educadoras en la sala de clases y esto podría influir a la hora de servir como modelos demostrativos de ejercicios físicos para sus estudiantes. Por otra parte, estudios previos han asociado la disminución de la potencia de salto con una mayor incidencia de enfermedades metabólicas, utilizándolo como un indicador de salud (31). Los valores promedios de vo2max obtenidos por las educadoras de párvulos

\section{TABLA 2}

Comparación entre grupos dado por IMC para las variables antropométricas, de composición corporal, metabólicas y de capacidad aeróbica, en relación a los valores de la OMS para mayores de 20 años (12).

Variables Normopeso $(n=19) \quad$ Sobrepeso $(n=19) \quad$ Obesidad $(n=8) \quad P$ valor

Variables antropométricas

$\begin{array}{lc}\text { Edad (años) } & 39,6 \pm 9,1 \\ \text { Peso (k) } & 61,6 \pm 7,3 \\ \text { Talla (m) } & 1,62 \pm 0,09\end{array}$

$38,5 \pm 9,3$

$68,3 \pm 5,4 a$

$1,57 \pm 0,05$

$41,5 \pm 6,5$

$79,3 \pm 8,2 b$

$1,57 \pm 0,08$

0,715

$0,000 * *$

0,089

Variables de composición corporal

Masa grasa (\%)

Masa magra (\%)

$31,6 \pm 4,7$

$64,6 \pm 4,9$

$35,7 \pm 5,2 \mathrm{a}$

$61,6 \pm 4,7$

Masa ósea (\%)

$3,5 \pm 0,4$

Tasa metabólica basal (kcal/dia)

$1257,2 \pm 63,7$

$3,3 \pm 0,3$

$1367,9 \pm 95,1 \mathrm{a}$

$41,9 \pm 3,2 b$
$55,1 \pm 3,1 a b$
$2,9 \pm 0,1 a b$
$1406,8 \pm 117,8 a$

$0,000^{* *}$

$0,000^{* *}$

$0,000^{* *}$

$0,000 * *$

Variables de capacidad física

Test de fuerza explosiva Squat jump

Altura alcanzada(cm)

$13,7 \pm 3,1$
$0,23 \pm 0,05$
$1691,1 \pm 512,4$

$14,4 \pm 4,0$

$0,22 \pm 0,07$

Altura alcanza/peso (cm/k)

$1691,1 \pm 512,4$

$1860,2 \pm 358,4$

Test de capacidad aeróbica UKK test

FC Inicio del Test UKK (Ipm)

FC Final del Test UKK (Ipm)

$\begin{array}{cc}76,1 \pm 8,8 & 83,2 \pm 9,6 \\ 155,4 \pm 15,6 & 160,0 \pm 17,5 \\ 18,6 \pm 0,9 & 18,7 \pm 1,1 \\ 28,9 \pm 2,9 & 26,6 \pm 4,3\end{array}$

$\begin{array}{cc}13,5 \pm 3,9 & 0,799 \\ 0,17 \pm 0,05 a & 0,075 \\ 2345,5 \pm 442,5 a b & 0,04^{* *}\end{array}$

$87,5 \pm 12,9 a$

$0,017^{*}$

$151,4 \pm 20,7 \quad 0,464$

$19,9 \pm 1,7 a b \quad 0,035^{*}$

$22,0 \pm 5,7 \mathrm{ab} \quad 0,001^{* *}$

Los valores están expresados en media desviación estándar. ANOVA, ${ }^{*} p<0,05 y{ }^{* *} p<0,001$ y análisis post hoc de Duncan.

aComparación en relación al grupo normopeso. ${ }^{\mathrm{b}} \mathrm{Comparación} \mathrm{en} \mathrm{relación} \mathrm{al} \mathrm{grupo} \mathrm{sobrepeso.}$ 
en el test UKK fueron de $26,7 \pm 4,7 \mathrm{ml} \bullet \mathrm{kg}-1 \bullet \mathrm{min}-1$. Además, las educadoras con obesidad presentaron valores promedio de vo 2 max menores a las educadoras con normopeso. Tanto el valor promedio de la muestra como el de cada grupo dividido por IMC fue menor de acuerdo a valores de referencia en mujeres sedentarias de rango de edad entre 35 a 44 años $(30,0 \pm 5,4 \mathrm{ml} \bullet \mathrm{kg}-1 \bullet \mathrm{min}-1)(32)$. Esto indicaría que las educadoras poseen una capacidad cardiorrespiratoria muy baja, lo que podría estar asociado a una baja práctica de ejercicio físico en la población en estudio y/o a deficientes hábitos de actividad, los cuales no fueron medidos. Otros estudios si han considerado estas variables y los resultados mostraron que sólo el $40 \%$ de las educadoras de párvulo consideraba el sobrepeso como un riesgo para la salud física. Por otro lado, las educadoras con obesidad pasaban más tiempo frente a un televisor o una pantalla y cuando comían lo hacían menos con sus familias y más frecuentemente mirando televisión que las educadoras con peso normal (5). El hecho que las educadoras, y en particular las que presentaron altos niveles de IMC, tuvieran bajas capacidades cardiorrespiratorias podría condicionar la intensidad y frecuencia con la que se realicen las actividades físicas en los jardines infantiles, lo que podría influir directamente en el nivel de actividad física de los niños que educan.

La importancia de un perfil nutricional normal y una buena condición física de educadoras de párvulo podría repercutir en la población infantil. Ma et al (2014) afirmaron que la educadora es un modelo a seguir en su labor educativa y cuando ellas son más activas, los niños son más activos (33). En relación a esto, de Craemer et al.(2014) realizaron un estudio a educadoras de párvulo, el cual reveló que el $41,2 \%$ presentaban un preocupante estado de sobrepeso; y por consiguiente, el riesgo de tener alumnos con sobrepeso en edades preescolar aumentaba aproximadamente 2,32 veces (34). En este sentido, un estudio reciente evaluó a asistentes de educadoras de párvulos, padres y alumnos, confirmando que existía una relación entre el IMC de los padres con los de sus hijos y entre el IMC de la asistentes de las educadoras y el IMC de los alumnos. Estos autores también mencionaron que los comportamientos saludables de las asistentes tenían un efecto duradero en los hábitos de vida de los niños de edad preescolar (20).

En la población infantil, los niveles de inactividad física son elevados y el patrón de actividad física en niños preescolares tiene una tendencia a ser sumamente reducido. Un estudio de Vásquez et al. (2005) comparó a niños asistentes a jardines infantiles con niños que no asisten, los resultados mostraron que no hubo diferencias en patrón de sedentarismo (35), lo cual nos podría estar indicando la baja capacidad de los jardines por aumentar el nivel de actividad física y promocionar estilos de vida saludable en los alumnos. Diversos autores han demostrado que la cantidad de actividad física realizada por los niños en jardines infantiles dependen directamente del jardín al que asistan (36), lo que hace referencia a la importancia del tipo de ambiente en el que se realicen las actividades (dentro o fuera de la sala), el equipamiento que se utiliza, el tamaño y color de los patios (37). Además, la evidencia plantea que la participación de modelos reales de estilos de vida saludable en educación es fundamental para combatir la obesidad y el sedentarismo (38).

Destacamos como una fortaleza del estudio la batería de evaluación nutricional, de composición corporal y condición física. Fue rápida, efectiva, económica, fácil de realizar y segura para quienes fueron evaluadas, no se presentaron abandonos durante la ejecución, ni se generaron efectos desfavorables posterior a esta. Por lo que podría servir de guía para un

TABLA 3

Correlación bi-variadas de Pearson (r) entre variables antropométricas, de composición corporal y capacidad física dado por IMC y \% masa grasa.

Variables

(r)

$-0,59 * *$

$-0,57^{* *}$

$0,63^{* *}$

$-0,18$

$-0,48^{* *}$

$0,39 * *$

$0,37^{*}$

0,05

$0,34^{*}$

$-0,59 * *$
\%Masa grasa

(r)

$-0.91^{* *}$

$-0,81^{* *}$

$0,39 * *$

$-0,98$

$-0,49 * *$

$0,47^{* *}$

0,21

0,02

$0,31^{*}$

$-0,43^{* *}$

$(n=46),{ }^{*} p<0,05 y{ }^{* *} p<0,001$ 
programa más extenso de evaluación física, nutricional y de condición física de educadoras de párvulo. Por otra parte, la comparación del IMC de estos resultados con valores de referencia nacional en relación a edad y género permite cuantificar el incremento de la incidencia de sobrepeso existente en este grupo específico de trabajadoras. Además, por primera vez en nuestro país se obtienen datos acerca de la condición nutricional y física de las educadoras de párvulo. La baja condición física y el IMC con tendencia al sobrepeso y obesidad podrían condicionar el actuar de la educadora y no ejercer correctamente su rol de modelo en estilos de vida saludable y práctica de actividad física.

Entre las limitaciones de nuestro estudio, se mencionan que se hizo difícil comparar nuestros resultados debido a la escasez de estudios similares. Por otra parte, el diseño de estudio limita la opción de obtener relaciones causales de las variables. El tamaño de nuestra muestra no permite extrapolar los resultados a la población de educadoras de párvulo de Chile, por la misma razón no se pudo dividir y analizar la muestra por rangos etarios. Tampoco se evaluó el impacto que pudo tener el IMC y condición física de las educadoras en el IMC y condición física de los niños que educan. Se recomiendan estudios más amplios y con un mejor diseño que puedan caracterizar más profundamente cual es el IMC, condición física y psicológica de este grupo de estudio, y como este impacta en la salud de los niños.

\section{CONCLUSIÓN}

La evaluación del IMC, la composición corporal y la condición física de las educadoras de párvulos del sistema municipal de la provincia del Bio-Bio mostró que un gran porcentaje de ellas presentan sobrepeso y obesidad, con altos porcentajes de grasa. Las educadoras con mayor IMC presentan peor condición física, aun cuando no sabemos si estos resultados se confirman en otras ciudades o países. En espera de futuros estudios, los resultados obtenidos podrían darnos una orientación de la actual condición nutricional y física de las educadoras de párvulo de la provincia del Bio-Bio.

\section{RESUMEN}

Objetivo: Describir el índice de masa corporal (IMC), composición corporal, la tasa metabólica basal, condición física y su relación en educadoras de párvulos chilenas. Sujetos y Métodos: 46 educadoras (edad $39,5 \pm 8,6$ ) de la provincia de Bio-Bio (Chile) se pesaron, midieron y se obtuvo su IMC. Se midió el porcentaje de masa grasa, magra, ósea, tasa metabólica basal, fuerza explosiva y capacidad cardiorrespiratoria. Resultados: Las educadoras tuvieron un IMC promedio de sobrepeso $(26,7 \pm 3,5)$ y un elevado porcentaje de grasa $(35,1 \pm 5,9)$ de acuerdo a referencias nacionales. El 58,7\% de ellas presentó sobrepeso y obesidad moderada. Cuando se comparó por IMC, las educadoras con obesidad presentaron en promedio un mayor porcentaje de masa grasa $(P=, 000)$, menor de masa magra $(P=, 000)$, demoraron más tiempo en terminar el UKK test $(P=, 035)$ y tuvieron un menor vo2max $(P=, 001)$ en comparación a las educadoras con normopeso. El IMC se asoció negativamente a los test de rendimiento físico. Conclusión: Las maestras con sobrepeso/obesidad presentaron más masa grasa, menos masa muscular y peores resultados en las pruebas de condición física. Condición preocupante pensando en su importante rol modelando hábitos de vida saludables en los prescolares.

Palabras clave: índice de masa corporal; composición corporal; aptitud física; docentes.
Agradecimientos: El autor Igor Cigarroa Cuevas fue apoyado por la beca de Perfeccionamiento Académico de la Universidad Santo Tomás (Chile) por el tiempo que demoró realizar esta investigación, además este trabajo fue apoyado por CONYCYT/BECA CHILE/PAI 72150035.

\section{BIBLIOGRAFÍA}

1. Lawrence RG. Framing obesity the evolution of news discourse on a public health issue. Harvlnt J Press-Pol 2004; 9(3): p. 56-75.

2. Ministerio de Salud de Chile. Pontificia Universidad Católica de Chile, Universidad Alberto Hurtado. Encuesta Nacional de Salud 2009-2010. Santiago, Chile. [Available:http://web.minsal.cl/portal/url/item/bcb03d7bc28b64dfe040010165012d23.pdf] [Found 22 de enero del 2015].

3. Hu FB, Li TY, Colditz GA, Willett WC, Manson JE. Television watching and other sedentary behaviors in relation to risk of obesity and type 2 diabetes mellitus in women. JAMA 2003; 289(14): 1785-91.

4. McAuley PA, Beavers KM. Contribution of cardiorespiratory fitness to the obesity paradox. Prog Cardiovasc Dis. 2014; 56(4): 434-40.

5. Hoffmann S, Tug S, Simon P. Obesity prevalence and unfavorable health risk behaviors among german kindergarten teachers: cross-sectional results of the kindergarten teacher health study. BMC Public Health 2013; 13(927):1-12.

6. Ministerio de Educación, Chile. Bases curriculares de la educación parvularia. Santiago, Maval Ltda 2005.

7. Kain J, Leyton B, Concha F, Salazar G, Lobos L. \&Vio F. Effect of counselling school teachers on healthy lifestyle on the impact of a program to reduce childhood obesity. Rev Med Chil. 2010;138(2): 181-7.

8. Monsalves-Álvarez M. Castro-Sepúlveda M, Zapata-Lamana $R$, Rosales-Soto $G$, Salazar $G$. Motor skills and nutritional status outcomes from a physical activity intervention in short breaks on preschool children conducted by their educators: a pilot study. Nutr Hosp 2015; 32(4):1575-80.

9. Brown WH. Effects of teacher-encouraged physical activity on preschool playgrounds. J Early Interv. 2009; 31(2): 126-45.

10. Ikeda T, Aoyagi O. Relationships between test characteristics and movement patterns, physical fitness, and measurement characteristics: Suggestions for developing new test items for 2-to 6-year-old children. Human Performance Measurement 2008;5:9-22.

11. Puche RC. Body Mass Index and the reasoning of an astronomer. Medicina (Buenos Aires) 2005; 65 (4): 361-5.

12. World Health Organization (WHO). Obesity: preventing and managing the global epidemic. Report of a WHO Consultation. WHO Technical Report Series 894. Geneva, Switzerland;1999. p. 6-13.

13. Jebb $S$, Cole $T$, Doman D, Murgatroyd $P$ \& Prentice $A$. Evaluation of the novel Tanita body-fat analyser to measure body composition by comparison with a four-compartment model. Brit J Nutr. 2000; 83: 115-22.

14. Markovic G, Dizdar D, Jukic I, Cardinale M. Reliability and factorial validity of squat and countermovement jump tests. J Strength Cond Res. 2004; 18(3): 551-5.

15. Castro-Sepulveda M, Monsalves M, Perez C. Relationship between jump height and anthropometric variables of cardiovascular risk in sedentary young. Rev Edu Fis. 2014; 30(3): 1-6.

16. Álvarez $C$, Ramírez $C$, Henríquez C, Castro M, Carrasco 
V \& Martínez C. Eight weeks of combined high intensity intermittent exercise normalized altered metabolic parameters in women. Rev Med Chil. 2014; 142(4):458-66.

17. OECD (2015), "Overweight and obesity among children", in Health at a Glance 2015: OECD Indicators, OECD Publishing, Paris.

18. Shulte PA, Wagner GR, Ostry A, Blanciforti LA, Cutlip RG, Krajna KM, et al. Work, obesity, and occupational safety and health. Am J Public Health 2007; 97(3):428-36.

19. Wardle J, Waller J, Yarvis MJ. Sex differences in the association of socioeconomic status with obesity. AM J Public Health 2002; 92(8):1299-304.

20. Hoffmann S, Tug S \& Simon P. Child-caregivers' body weight and habitual physical activity status is associated with overweight in kindergartners, BMC Public Health 2014;14(822):1-13.

21. Mei Z, Grummer-Strawn LM, Pietrobelli A, Goulding A, Goran MI, Dietz WH. Validity of body mass index compared with other body-composition screening indexes for the assessment of body fatness in children and adolescents. Am J Clin Nutr. 2002; 75(6):978-85.

22. Moreno, M. Definition and classification of obesity. Rev Med Clin Condes 2012; 23(2): 124-8.

23. Kain J, Uauy R, Concha F, Leyton B, Bustos N, Salazar G, Lobos L \&Vio F. School-Based Obesity Prevention Interventions for Chilean Children During the Past Decades: Lessons Learned. Adv Nutr Rev J. 2012;3(4):616-21.

24. Hernández $R$, Martínez $B$, Pérez DS, Navas CS \&Martínez J. Comparative study of body composition measures by dual $X$-ray absortiometry, bioimpedance and skinfolds in women. An R Acad Nac Farm. 2010;76(2):209-22.

25. Rosado LE, Bressan J, D'Andrea CL \& Vogel CE. Body composition and energy metabolism in women with excess body weight. Anales Sis San Navarra 2010;(33)2:155-65.

26. Ortega FB, Ruiz JR, Castillo MJ, Sjöström M. Physical fitness in childhood and adolescence: a powerful marker of health. Int J Obes (Lond). 2008;32:1-11.

27. LaMonte MJ, Blair SN. Physical activity, cardiorespiratory fitness, and adiposity: contributions to disease risk. Curr Opin Clin Nutr Metab Care. 2006 Sep;9(5):540-6.

28. Ortega FB, Ruiz JR, Hurtig-Wennlof A, Vicente-Rodriguez $G$, Rizzo NS, Castillo MJ, et al. Cardiovascular fitness modifies the associations between physical activity and abdominal adiposity in children and adolescents. The European Youth Heart Study. Br J Sports Med. 2010;44:256-62.

29. Ortega FB, Ruiz JR, Castillo MJ. Physical activity, physical fitness, and overweight in children and adolescents: evidence from epidemiologic studies. Endocrinol Nutr. 2013;60(8):458-69.

30. Ardoy, D., Fernández-Rodríguez, J., Ruiz, J., Chillón, P., España-Romero, V., Castillo, M., \& Ortega, F. Improving Physical Fitness in Adolescents Through a SchoolBased Intervention: the EDUFIT Study. Rev Esp Cardiol. 2011;64(6):484-91.

31. Newman $B$, Varant $K$, Marjolein $V$, Eleanor $M$, Simonsick $H$, Goodpaster S, Kritchevsky Frances A. Tylavsky S, Rubin M, Harris T. Strength, but not muscle mass, is associated with mortality in the health, aging and body composition study cohort. J Gerontol A Biol Sci Med Sci. 2006; 61: (1) 72-7.

32. Herdy $A H$, Uhlendorf $D$. Reference values for cardiopulmonary exercise testing for sedentary and active men and women. Ar Bra Cardiol. 2010; 96(1): 54-9.

33. Ma K, Le Mare L \& Gurd JB. Classroom-based highintensity interval activity improves off-task behaviour in primary school students. Appl Physiol Nutr Metab. 2014;39(12)1332-7.

34. De Craemer M, De Decker E, De Bourdeaudhuij I, Deforche $B$, Vereecken $C$, Duvinage $K$, Grammatikaki $E$, lotova $V$, Fernández A, Zych C, Manios Y \&Cardon G. Physical activity and beverage consumption in preschoolers: focus groups with parents and teachers. BMC Public Health 2013; 27;(13):278.

35. Vásquez F, Salazar G. Physical activity pattern a group of obese preschool children, attending daycare centers (JUNJI), evaluated by a movement sensor. Rev Chil Nutr. 2005; 32, (2):1-12.

36. Pate $R$. Directly observed physical activity levels in preschool children. J Sch Health 2008; 78(8): 438-44.

37. Hannon JC. Brown B. Increasing preschoolers' physical activity intensities: an activity-friendly preschool playground intervention. Prev Med. 2008; 46(6): p. 532-6.

38. Davó M, Gil G, Vives C, Álvarez D, La Parra D. Research on health education and promotion in Spanish nursery and primary schools. A systematic review of studies published between 1995 and 2005. Gac Sanit. 2008; 22(1): 58-64. 